(Aus dem physiol. Institui aer tierärztl. Hochschule in Stuttgart.)

\title{
Der Druck im Cavum pleurae des Pferdes.
}

Von

R. Bendele, Backnang.

(Mit 5 Textfiguren.)

Zuntz ${ }^{1}$ ) hat bei seinen respiratorisehen Stoffwechseluntersuchungen des Pferdes gezeigt, dass die Atemgrösse des Pferdes, das von der Ruhe zur Arbeit übergeht, sofort um das $15-20$ fache ohne irgendwelche Atemnot steigen kann. Es drückt sich damit eine so grosse Anpassung des Pferdes an Bewegung und äussere Arbeit aus, dass es sich wohl verlohnt, den Bedingungen zu dieser Anpassung nachzugehen.

Es liegt nahe, die Anpassung in Beziehung zu dem luftverdünnten Raume in der Pleurahöhle zu bringen, zumal eine ganze Anzabl von Forsehern die Wirkung desselben für die Atmung selbst und sodann für die Zirkulation des Blutes und der Lymphe nachgewiesen haben. Gerade für das Pferd aber liegen direkte Bestimmungen des. Druckes im Cavum pleurae nicht vor, worauf $\mathrm{Gmelin}^{2}$ ) bereits aufmerksam gemacht hat. Indirekte Messungen mit Hilfe der Elastizität der Lungen hat Sussdorf $\mathrm{f}^{3}$ ) vorgenommen; allein es können diese keine genauen Resultate haben, wie unten gezeigt werden soll. Im folgenden ist daher versucht worden, mit Hilfe direkter Methoden am Jebenden Pferd während ruhiger und ge-

1) Zuntz und Hagemann, Untersuchungen über den Stoffwechsel des Pferdes bei Ruhe und Arbeit. 1898. - Zuntz, Praktische Folgerungen aus den beim Arbeitspferd ausgeführten Stoffwechseluntersuchungen. Zeitschr. f. Veterinärkunde, 8. Jahrg., Nr. 7 S. 293.

2) Gmelin, Die Atmung. - Ellenberger-Scheunert, Lehrbuch der vergleichenden Physiologie der Haussängetiere S. 138. 1910.

3) Sussdorf, Die Atmung. In Filenberger, Handbuch der vergleichenden Physiologie der Haussäugetiere, I. T., S. 630. 1890. 
steigerter Atmung die Grösse des Drucks in der Pleurahöhle zu ermitteln.

Der erste, welcher sich mit den Druckschwankungen in der Pleurahöhle befasste, war $\mathrm{Ludwig}{ }^{1}$ ). Allein praktische Zablenwerte konnten bei der primitiven Art der Untersuchungsmethode nicht gewonnen werden. Bekannt sind die Versuche von Donders's), welche die Grundlagen für jede weitere Erforsehung der Frage bilden.

Seine Methode war eine indirekte, indem er an der Leiche durch ein in die Trachea eingeführtes Manometer die Kraft mass, mit welcher sich die Lunge bei Eröffnung des Thorax zusammenzog. Der dabei gefundene positive Druck entspricht dem negativen während der Dauer der Exspiration. Donders fand beim Menschen einen Druck von - 30 bis $-70 \mathrm{~mm}$ Wasser. Die Elastizität völlig gesunder Lungen glaubt er auf $-80 \mathrm{~mm}$ Wasser angeben zu dürfen. Da ferner Donders den jeweiligen Spannungsgrad der Lungen nicht zu messen vermochte, so fehlen Angaben, aus denen die durchsehnittliche Grösse des intrapleuralen Druckes bei der Inspiration zu berechnen wäre. Auf irgendwelche Muskelwirkung bei der Exspiration ist dabei nicht Rücksicht genommen. Fine solche findet aber, wie Gmelin ${ }^{3}$ ) zeigt, beim Pferd, sobald es nicht oberflächlich atmet, statt. Donders Untersuchungsresultate führten dahin:

1. dass bei normaler ruhiger Atmung, wobei das Ausatmen passiv ohne Muskelwirkung durch die Elastizität der Lungen geschieht, die Pleurafläche der Lunge und alle ausserhalb der Lunge im Thorax liegenden Organe unter einem Druck stehen, der um so viel hinter dem atmosphärisehen zurückbleibt, als die Elastizität der Lungen ausmacht;

2. dass die elastische Kraft der Lungen während der Inspiration zunimmt, und demnach die Saugkraft der Lungen während der Inspiration grösser, der negative Druck somit ausgesprochener ist als während der Exspiration.

Heynsius ${ }^{4}$ ) hat eingehende Untersuchungen über die Grösse

1) C. Ludwig, Kenntnisse über den Zusammenhang zwischen den Respirationsbewegungen und dem Kreislauf. Arch. f. Anat. 1847 S. 248.

2) Donders, Beiträge zum Mechanismus der Respiration und Zirkulation im gesunden und kranken Zustande. Zeitschr. f. ration. Medizin Bd. 2 S. 287. 1853.

3) Gmelin, l. c.

4) Heynsius, Die Grösse des negativen Drucks im Thorax beim ruhigen Atmen. Pfiùger's Arch. Bd. 29 S. 265. 1882. 
des negativen Drucks im Thorax beim ruhigen Atmen angestellt. Auch seine Untersuchungen sind indirekt, obschon, wie er sagt, der kürzeste Weg, welcher sofort züm Ziele führen würde, natürlich der wäre, dass man die Grösse des negativen Drucks bei normaler Inspiration und Exspiration am lebenden Tiere durch ein mit der Pleurafläche verbundenes Manometer bestimmte. Diese direkte Methode hat nach Ansicht von Heynsius den grossen Übelstand, dass man wenig Aussicht hat, auf diese Weise die Verhältnisse bei normaler ruhiger Atmung kennen zu Jernen, da diese durch Öffnung des Thorax und Einführung einer Kanüle beträchtlich gestört wird. Er sucht daher den intrathorakalen Druck aus der Elastizität des Lungengewebes bei verschiedenen Expansionsgraden zu ermitteln. Bei seinen Versuchen ging er von dem Gesichtspunkt aus, dass brauchbare Resultate nicht durch positiven Druck von seiten der Trachea, sondern nur durch Verminderung des Drucks auf der Pleurafläche zu erzielen seien. Deshalb liess er an der Leiche das Zwerchfell, nachdem die Trachea mit einem entsprechenden Manometer verbunden war, so lange herabziehen, bis die erforderliche Luftmenge in die Lungen eingesogen war. Wurde nun das Zwerchfell freigelassen, und der entsprechende Hahn des Manometers geöfnet, so liess sich die elastische Kraft der Lungen unter diesen Umständen ablesen. Heynsius fand bei Hunden von weniger als $10 \mathrm{~kg}$ Gewicht den negativen Druck im Thorax bei Inspiration im Mittel $-7 \mathrm{~mm} \mathrm{Hg}$, bei Exspiration im Mittel $-4 \mathrm{~mm} \mathrm{Hg}$, Druck differenz $-3 \mathrm{~mm} \mathrm{Hg}$. Bei Hunden über $10 \mathrm{~kg}$ ergab sich bei Inspiration im Mittel - 7,5 $\mathrm{mm} \mathrm{Ho}$, bei Exspiration - 4,0 $\mathrm{mm} \mathrm{Hg}$, Druckdifferenz im Mittel $-3,5 \mathrm{~mm}$ Hg. Bei Kaninchen beträgt der negative Druck bei Inspiration - 7,6 mm $\mathrm{Hg}$, bei Exspiration $-2,5 \mathrm{~mm} \mathrm{Hg}$; Druckdifferenz im Mittel $-5,1 \mathrm{~mm} \mathrm{Hg}$. Heynsius sagt, der beim Loslassen des Diaphragmas beobachtete positive Druck in der Trachea drückt innerhalb der angegebenen Grenzen rein und ausschliesslich die elastische Kraft der Lungen aus und gewährt demnach einen reinen Massstab des in den Pleurahöhlen herrschenden negativen Drucks, d. h. der Saugkraft des Thorax.

Sussdorf ${ }^{1}$ ) hat nach dem Verfahren von Donders durch Einführen eines Manometers in die Trachea und Eröffnen der Pleurahöhlen den negativen Druck im Cavum pleurae des Pferdes ge-

1) Sussdorf, l. c. 
messen und gibt ihn auf $-7 \mathrm{~mm} \mathrm{Hg}$ im Stadium der höchsten Exspiration, auf ca. $-28 \mathrm{~mm} \mathrm{Hg}$ in dem der tiefsten Inspiration an.

Luciani') und Rosenthal ${ }^{2}$ ) baben den negativen Druck dadurch gemessen, dass sie eine Sonde mit weitem Volumen (Ösophagusexplorateur) in den Ösophagus einführten. Verbindet man diese Sonde mit einem Manometer unter Ausschluss des atmosphärischen Drucks, so zeigt das Manometer den negativen Druck direkt an. Er beträgt, auf diese Weise gemessen, nach Rosenthal bei Kaninchen bis $-40 \mathrm{~mm}$ Wasser. Die Werte des negativen Druckes mittelst der Ösophagussonde fallen jedoch stets zu niedrig ans, da die Ösophaguswand und das sie umschliessende Bindegewebe des Mediastinums einen gewissen Widerstand ausüben. Um das Mass dieses Widerstandes fällt der negative Druck, der mit geschlossener Ösophagussonde im Schlund gefunden wird, zu gering aus.

Bestimmungen über die Druckschwankungen im Thorax durch direktes Einführen von entsprechenden Kanülen in die Brusthöhle haben Aron, Büdingen, Van der Brugh gemacht. Praktische Resultate kann man mit Kanülen nur dann erhalten,

1. wenn sie sich in den Raum zwischen Brustwand und Lunge ohne Verletzung der letzteren und ohne Herstellung eines Pneumothorax einführen lassen;

2. wenn ein Verschluss der inneren Mündung der Kanüle bei den Bewegungen der Lunge nicht stattfinden kann, und

3. wenn das Instrument, das mit einem Manometer oder Schreibapparat verbunden ist, trotz Hebung und Senkung der Brustwand in unveränderter Lage zur Lunge fixiert ist.

$\operatorname{Aron}{ }^{3}$ ) hat an einigen kranken und einem gesunden Mensehen den negativen Druck direkt gemessen und hat dazu einen geschärften Trokar in die Brusthöhle eingeführt. Die Verwendung eines solchen halte ich jedoch schon aus dem Grunde für bedenklich, da bei Verwendung eines nicht geschützten Troikars die Lunge sehr leicht verletzt wird. Bei gesunden Menschen gibt er die Werte des intrapleuralen Drucks auf $-5,09 \mathrm{~mm} \mathrm{Hg}$ und $-4,23 \mathrm{~mm} \mathrm{Hg}$ an.

1) Luciani, Physiologie des Menschen. Übersetzt und bearbeitet von Baglioni, Winterstein und Verworn. Bd. I S. 356. 1905.

2) Rosenthal in Hermann, Handbuch der Physiologie des Menschen. 2. T., Bd. 10 S. 226. 1882, und Arch. f. Physiol. Suppl. 1880 S. 34.

3) Aron, Der intrapleurale Druck beim lebenden, gesunden Menschen. Virchow's Arch. Bd. 126 s. 519. 1891; Bd. 160 S. 228.1900. 
Büdingen ${ }^{1}$ ) hat seine Untersuchungen an narkotisierten Hunden und Kaninchen vorgenommen. Auf den zu den Untersuchungen verwendeten Thoraxdruckmesser wird im späteren zurückzukommen sein. Die Resultate seiner Untersuchungen decken sich beinahe mit den von Heynsius auf indirekte Weise gefundenen. Er stellte den negativen Druck bei Hunden zwischen

-48 und $-72 \mathrm{~mm}$ Wasser bei Exspiration,

$-81,-178, "$ "Inspiration

fest. Die Konstanz dieser Zahlen erleidet wesentliche Änderungen unter sonst normalen Verhältnissen dureh Verengerung oder Verschluss innerhalb der oberen Luftwege, z. B. forcierter Exspiration und gleichzeitig geschlossener Glottis, so dass hierbei selbst positiver Thoraxdruck zustande kommen kann. Positive Werte des thorakalen Druckes sind nur möglich, wenn der Alveolardruck den atmosphärischen erheblich übersteigt und dies kann wiederum nur der Fall sein, wenn dem Ausströmen der Luft bedeutende Hindernisse entgegenstehen.

Van der Brugh ${ }^{2}$ ) bat unter Einthoven's Leitung den intrapleuralen Druck gemessen. Er ging von dem Gesichtspunkte aus, dass beim Einführen einer Kanüle in die Brusthöhle immer Luft mitströmt, was eine Volumverringerung der Lunge zur Folge hat. Dadurch wird ihre elastische Spannung und somit auch der negative intrapleurale Druck kleiner, deshalb hat er gleichzeitig Druck und Volummessungen vorgenommen. Man bringt zu diesem Zwecke ein gemessenes Quantum Luft in die Pleurahöhle eines Hundes und misst hierauf den intrapleuralen Druck. Durch allmähliches Hinzufügen weiterer Quantitäten Luft werden die Veränderungen beobachtet, die der intrapleurale Druck erleidet. Sind die Drucke bekannt, die in der Pleurahöhle herrsehen, wenn dieselbe so und so viel Kubikzentimeter Luft enthält, so lässt sich leicht ermitteln, wie hoch der Druck sein wird, wenn gar keine Luft mehr vorhanden ist. Die zu diesen Versuchen verwendete Pleurakanüle ist eine gerade Messingröhre, die an einem Ende durch einen Hahn

1) Büdingen, Experimentelle Untersuchungen der normalen und pathologisch beeinflussten Druckschwankungen im Brustkorb. Arch. f. exper. Pathol. Bd. 39 S, 245. 1897.

2) van der Brugh, Über eine Methode zur Messung des intrapleuralen Drucks. Pflüger's Arch. Bd. 82 . S. 591. 1900. 
verschlossen werden kann, während das andere von einem Bügel überbrückte Ende stets offen bleibt und zwischen zwei Rippen in die Pleurahöhle nach einem vorangegangenen Einschnitt eingeführt wird. Van der Brugh fand den negativen Druck bei der Inspiration nicht grösser als -80 , höchstens $-140 \mathrm{~mm}$ Wasser. Die Heynsius'schen Werte sind wesentlich kleiner als die von Van der Brugh; er glaubt dies dem Umstande zuschreiben zu müssen, dass Heynsius den intrapleuralen Druck nach dem Tode der Versuchstiere gemessen hat, während van der Brugh an lebenden Hunden seine Messungen vornahm. Van der Brugh hat auch nachgewiesen, dass tatsächlich der intrapleurale Druck sofort nach dem Tode abnimmt und einige Zeit nachher ganz versehwunden ist.

Vergleichende Versuche über den intraabdominalen und intrathorakalen Druck sind in der Arbeit von Winkler ${ }^{1}$ ) niedergelegt. Wie bei der Feststellung des intrathorakalen Drucks, so spielt auch beim intraabdominalen Druck die Stellung des Zwerchfells eine wichtige Rolle, derart, dass mit dem Niedergehen des Zwerchfells der Abdominaldruck sich hebt, und dass mit dem Hinaufsteigen bei $\mathrm{Zu}$ sammenfallen der Lunge der Abdominaldruck wieder sinkt.

Bevor ich zu meinen eigenen Versuchen übergehe, soll aus dem Vorhergehenden zusammengefasst werden:

1. am lebenden Pferde sind bis jetzt Messungsversuche zur Feststellung des negativen Drucks im Cavum pleurae nicht gemacht worden;

2. die an der Leiche gewonnenen Resultate sind nicht genau, weil der negative Druck nach dem Tode sofort kleiner wird und nach und nach ganz verschwindet. Zudem sind die dabei stets möglichen Muskelwirkungen unberücksichtigt;

3. über das Verhalten des negativen Drucks beim Übergang vom ruhigen zum gesteigerten und von diesem wiederum zum ruhigen Atmen ist nichts bekannt.

Die im folgenden beschriebenen Versuche fanden an nicht narkotisierten Pferden statt. Die Pferde wurden einige Zeit vor dem Versuch tracheotomiert und mit der Trendelenburg'schen Kanüle, wie sie auch Zuntz bei seinen Untersuchungen verwendete, ausgestattet. Es ist bekannt, dass Pferde sich aus einem

1) Winkler, Untersuchungen über die Beziehungen des Abdominaldrucks zur Respiration. Pflüger's Arch. Bd. 98. S. 163. 1908. 
solchen Tracheotubus nichts machen und ihn lange tragen; ohne irgendwelche Atmungsstörung zu zeigen. Angesichts der zu erwartenden erheblichen Druckdifferenzen bei angestrengter Atmung wurde stets auf absolut festen und zuverlässigen Sitz der Trachealkanüle Bedacht genommen. Während des Versuches standen die Pferde in einem sogenannten Notstand in natürlicher Körperhaltung. Möglichst dicht dabei, doch geschützt gegen etwaige Unruhe des Pferdes stand das Kymographion unit den Registriervorrichtungen.

Auf die Einführung des übrigens dünnen Thoraxdruckmessers reagierten die Pferde kaum bzw. gar nicht, wenn noch die Einstichstelle schwach kokainisiert war. Als Thoraxdruckmesser diente der Büdingen'sche, der etwas abgeändert wurde. Der Apparat besteht aus einem geschärften Troikar, in dessen Inneres ein hohler Bolzen eingepasst ist. Dieser ist an seinem freien Ende ausgiebig gefenstert, das andere Ende ist mit einer Feder verbunden, die den Bolzen in dern Augenblick vorschnellt, in welchem er in das Cavum pleurae eindringt. Der Hohlraum des Bolzens setzt sich fort in einen dicht schliessenden Hahn, der mit einem Manometer oder Schreibapparat in Verbindung gesetzt werden kann. Aussen auf dem Troikar ist eine ca. $4 \mathrm{~cm}$ im Durchmesser $=$ dem ungefähren Durchmesser eines Zwischenrippenraumes beim Pferde) betragende verstellbare Seheibe aufgepasst. Diese ist auf ihrer dem Brustkorb zugekehrten Fläche konkav und wird mit Wachssalbe gefullt; sie hat den Zweck, sobald der Apparat in die Pleurahöhle eingedrungen ist, ringsum den Stichkanal abzudichten. Die Vorzüge dieses Thoraxdruckmessers bestehen darin, dass er sich, da er die Form eines geschärften Troikars hat, leicht in die Brusthöhle einführen lässt. Während der Dauer des Einführens wird der Bolzen dureh den mechanischen Widerstand der Weichteile (Muskeln, Pleura) zurückgedrängt, schnellt jedoch sofort vor, wenn dieser Widerstand aufhört. Da der Bolzen an seinem oberen Ende abgerundet ist, so kann er selbst weiches und rissiges Gewebe wie die Lunge nicht verletzen, sondern er vermag dieselbe nur zurückzudrängen. Wird das Instrument mit einiger Vorsicht gehandhabt, so ist es schlechterdings unmöglich, einen Pneumothorax zu erzeugen, da erstens der Stichkanal klein ist, und die Muskulatur sofort den Kanal zu schliessen sucht, und da zweitens der Apparat sowohl bei den inspiratorischen, wie exspiratorischen Bewegungen des Brustkorbes in seiner Lage sich erhalten lässt. 
Bei den Versuchen wurde der Thoraxdruckmesser durch einen Druckschlauch von $3 \mathrm{~mm}$ Lichtweite mit einem Hürthle'schen Federmanometer verbunden, das die gegebenen Kurven auf ein Kymographion aufzeichnete. Die Schlauchlänge wurde so kurz wie möglich genommen, konnte aber, um Beschädigungen der Apparate bei etwaiger Unruhe des Tieres zu verhüten, nicht kürzer als $1-1,25 \mathrm{~m}$ gewählt werden. Die Kalibrierung der Kurven erfolgte sofort im Anschluss an den Versuch unter Benützung jeweils desselben Federmanometers und derselben Sehlauchleitung mittelst eines Hg-Aichmanometers. Zur gleichzeitigen Feststellung des intrathorakalen und intrapulmonalen Drucks wurde der oben erwähnte Tracheotubus mit einem weit gebohrten, für diese Zwecke von Professor Gmel in besonders konstruierten Dreiweghahn, an welchem seitlich ein Tubulus war, verbunden. Durch diesen Hahn liess sich je nach seiner Stellung Dyspnoë verschiedenen Grades erzeugen. Der seitliche Tubulus des Dreiweghahnes war mit einem zweiten, dem erst erwähnten, ganz gleich konstruierten Hürthle'schen Federmanometer zur Aufzeichnung des intrapulmonalen Drucks verbunden. Beide Manometer zeichneten gleichzeitig und gleichsinnig die Kurven des intra- und extrapulmonalen Drucks auf die rotierende Trommel.

Die ganze Versuchsanordnung wurde zunächst an mehreren Pferden einer Vorprobe unterzogen und dabei geprüft:

1. ob der Thoraxdruckmesser, ohne erhebliche Störungen in der Respiration hervorzurufen, und ob er besonders ohne vorbergegangene Narkose eingeführt werden kann;

2. ob durch das eingeführte Instrument Verletzungen der Lunge erzeugt werden. Fanden solche statt, so musste der Versuch ausscheiden. Die Feststellung fand jedesmal am frisch getöteten Tiere statt und wurde dadurch erleichtert, dass die Pferde meist in direktem Anschluss an den physiologischen Versuch für anatomische Zwecke getötet wurden ${ }^{1}$ );

3. ob durch den Thoraxdruckmesser, nachdem er mit einem Manometer oder Schreibapparat verbunden wurde, die verlangten Druckverhältnisse im Thorax sich feststellen liessen.

1) Dem Vorstand des anatomischen Instituts Herrn Direktor Dr. v. Suss dorf sèi auch an dieser Stelle der ergebenste Dank für die freundliche Überlassung der Anatomiepferde zu meinen Versuchen ausgesprochen. 
Diese - Voruntersuchungen hatten in allen Punkten ein für die weitere Arbeit günstiges Ergebnis.

Zunächst seien etliche Versuche angeführt, bei welchen der intrathorakale Druck allein gemessen wurde.

\section{Versuch I.}

15. Nov. 1909, vormittags 9 Uhr. Fohlen, 9 Monate, braun, Wallach. Atmung etwas frequent.

Intrathorakaler Druck (Inspiration) $-45 \mathrm{~mm} \mathrm{Hg}$. Das Fohlen wiehert wiederholt. Dabei lässt sich bei den dem Wiehern vorausgehenden, tiefen Inspirationen eine Zunahme des negativen Drucks auf $-62 \mathrm{~mm}$ feststellen. Bei der Exspiration wird der negative Druck in kürzester Zeit (ca. 1/6 Sekusde) positiv und erreicht eine Höhe von ca. $+65 \mathrm{~mm}$. Im ganzen bleibt er etwa 1,5 Sekunden positiv und sinkt dann auf den alten Wert von $-45 \mathrm{~mm} \mathrm{Hg}$ herab (Fig. $1 \mathrm{a}$ ).

15. November, nachmittags $4 \mathrm{Uhr}$, $3^{1 / 2}$ Stunden nach dem Töten, Feststellung des Drucks auf indirektem Wege durch Trachealkanüle und Wassermanometer: nach Eröffnung des Thorax +8 mm Wasser. Kadaver stark gasig aufgetrieben. Lunge selbst unverletzt. Es findet somit post mortem eine rasche Abnahme des negativen Drucks statt. Dadurch ist erwiesen, dass eine indirekte Druckmessung keine zuverlässigen Resultate liefert.

\section{Versuch II.}

23. Nov. 1909, vormittags $9 \mathrm{Uhr}$. Stute hellbraun, 20 Jahre alt. Das Pferd wird tracheotomiert. Die Atmung ist änsserst oberflächlich und sehṛ ruhig,

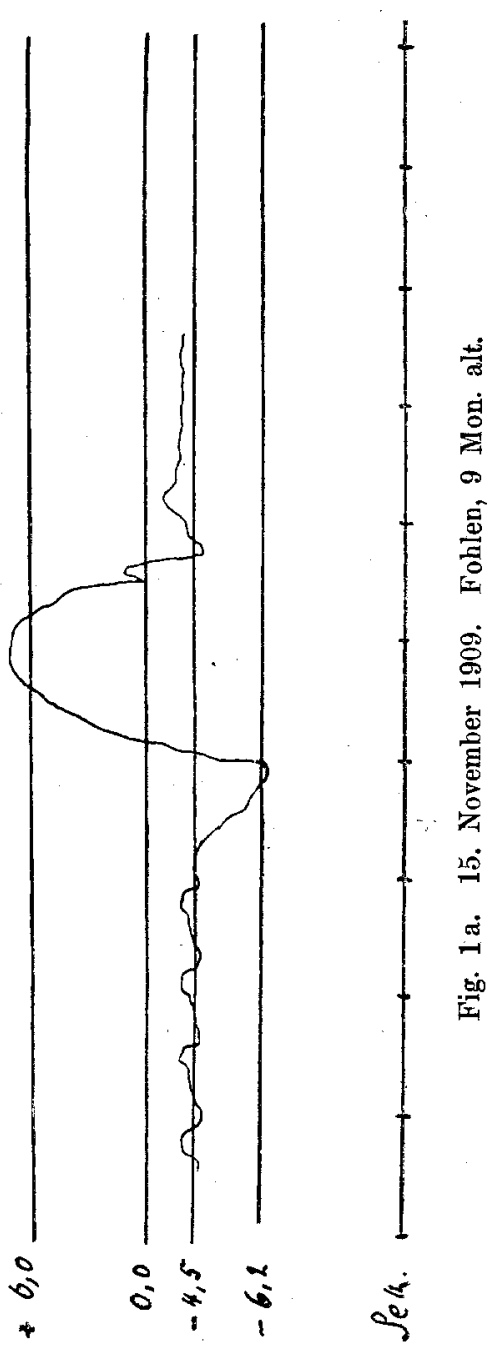
bei diesem Versuche wird zur Messung des intrapulmonalen Druckes ein Glycerinmanometer, auf dem ein Sclwimmer mit einer Schreibfeder sich befindet, verwendet.

Resultat: Intrathorakaler Druck $-60 \mathrm{~mm} \mathrm{Hg}$.

Besichtigung post mortem: Lunge unverletzt; allein rechte Lunge infolge alter Pneumonie herdweise hepatisiert. 
26. November 1909, 3 Uhr nachmittags. Dunkelbranner Wallach, 14 Jahre alt. Tracheotomiert. Einführen der Thoraxkanüle links im 7. Interkostalraum. Bei offener Trachea intrathorakaler Druck $-50 \mathrm{~mm} \mathrm{Hg.} \mathrm{Hieran} \mathrm{ändert} \mathrm{sich} \mathrm{bei}$ unvollständigem Verschluss der Trachealkanüle wenig. Wird diese im Moment der Einatmung vollständig geschlossen, so nimmt der negative Druck sofort zu und beträgt $-150 \mathrm{~mm} \mathrm{Hg}$.

Nach ca. 15 Sekunden beträgt er $-200 \mathrm{~mm}$ Hg, nach weiteren 40 Sekunden $-600 \mathrm{~mm} \mathrm{Hg}$; hochgradige Dyspnöe.

Nach Öfinen der Trachealkanüle nähert sich der negative Druck ganz allmählich seinem Ausgangswert und erreicht denselben nach ca. 80 Sekunden. Dieses Verhalten ist ein konstantes; denn es zeigt sich jedesmal nach Verschluss der Trachealkanüle.

\section{Versuche unter gleichzeitiger Registrierung des intrathorakalen und intrapulmonalen Drucks.}

\section{Versuch III.}

6. Dezember 1909. Fohlen 3/4 Jahr alt, Wallach, tracheotomiert, Bei Beginn des Versuchs intrathorakaler Druck - $50 \mathrm{~mm} \mathrm{Hg.} \mathrm{Mit} \mathrm{zunehmender} \mathrm{Ver-}$ tiefung der Atmung nach Verschluss des Dreiweghahns Zunahme des intrathorakalen Drucks auf $-100,-200,-300,-400,-500 \mathrm{~mm} \mathrm{Hg}$, ein Tiefstand, der in 33 Sekunden erreicht ist. Beim Öffnen des Hahns fällt der negative Druck sofort auf $-100 \mathrm{~mm} \mathrm{Hg}$ und bleibt längere Zeit auf dieser Höhe; erst allmählich stellt er sich auf die alte Höhe von $-50 \mathrm{~mm} \mathrm{Hg}$ ein.

Der gleichzeitig aufgezeichnete Pulmonaldruck bleibt hinter dem intrathorakalen stets um ca. $100 \mathrm{~mm} \mathrm{Hg}$ zurúck; sein maximaler Pluswert beträgt ca. +70 bis $+80 \mathrm{~mm} \mathrm{Hg}$, sein maximaler Minuswert ca. $-400 \mathrm{~mm} \mathrm{Hg} \mathrm{(Fig.} \mathrm{1).}$

Nach der Tötung des Fohlens wird auf indirektem Weg der negative Druck 3 Stunden post mortem gemessen. Er beträgt $-45 \mathrm{~mm}$ Wasser rechts, $-50 \mathrm{~mm}$ Wasser links.

\section{Versuch IV.}

13. Dezember 1909. Altes Anatomiepferd, Wallach, tracheotomiert. Intrathorakaler Druck bei offener Trachea -100 bis $-150 \mathrm{~mm} \mathrm{Hg}$ während der Inspiration, - $70 \mathrm{~mm} \mathrm{Hg}$ während der Exspiration.

Bei Verschluss der Trachea: Intrathorakaler Druck - $170 \mathrm{~mm} \mathrm{Hg}$; bei Inspiration, - $100 \mathrm{~mm} \mathrm{Hg}$ bei Exspiration.

Intrapulmonaler Druck $-90 \mathrm{~mm} \mathrm{Hg}$ bei Inspiration, $+50 . \mathrm{mm} \mathrm{Hg}$ bei Exspiration. Im Verlauf der Absperrung beträgt der intrathorakale Druck - $200 \mathrm{~mm} \mathrm{Hg}$, der intrapulmonale Druck $-120 \mathrm{~mm} \mathrm{Hg}$, und schlägt bei Exspiration um in positiv $+40 \mathrm{~mm} \mathrm{Hg}$.

Nach Öffnen des Absperrhahns sinkt der negative Druck zunächst auf $-85 \mathrm{~mm} \mathrm{Hg}$ and dann allmählich auf $-80 \mathrm{~mm} \mathrm{Hg}$. Der intrapulmonale Druck fällt nach Öffnen des Absperrhahns beinahe mit der Abszisse zusammen.

Eine Wiederholung der Versuche gibt stets gleiche Resultate, ferner bestätigt sich durch unsere direkten Messungen "die 


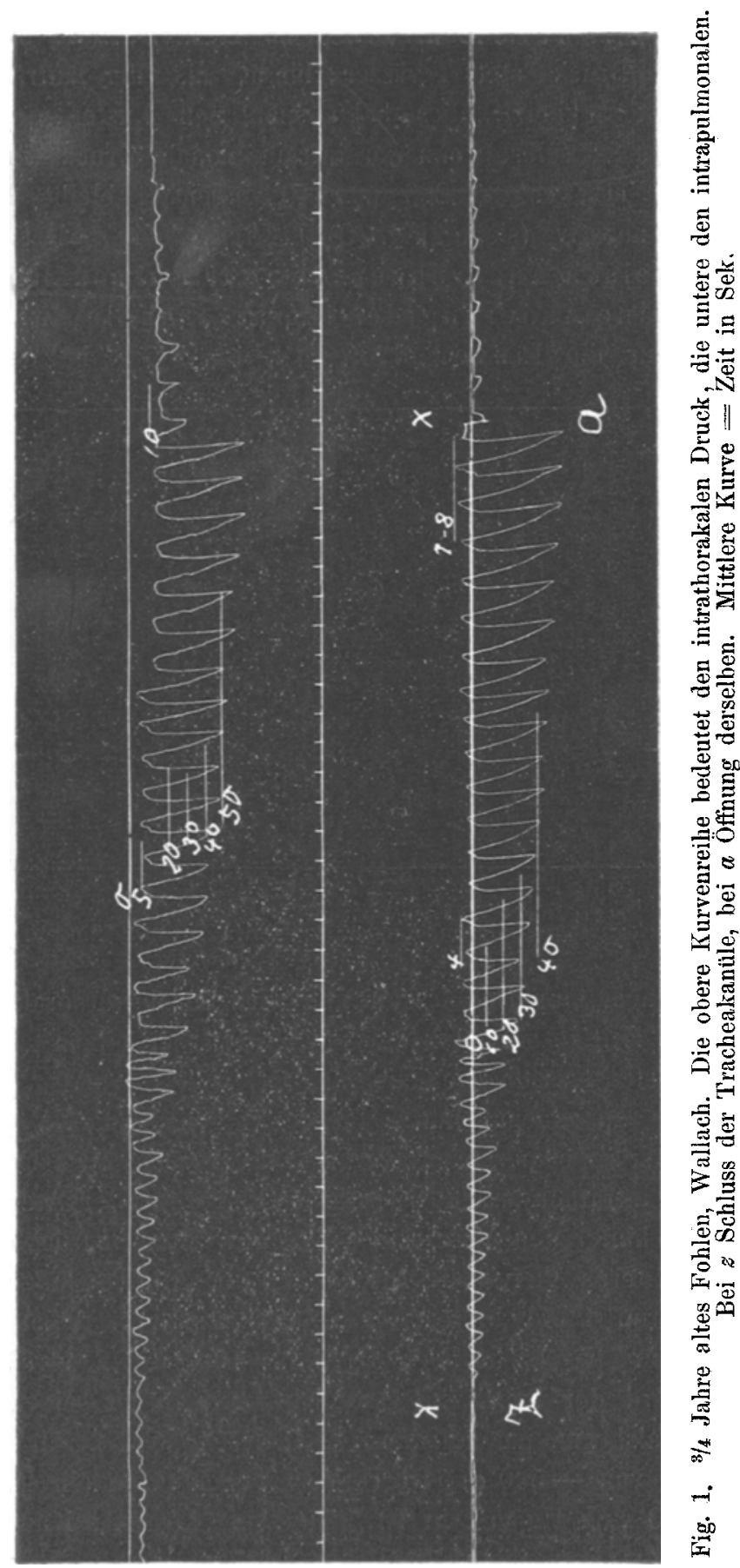


Angabe ven Ewald und van der Brugh, dass der intrapulmonale Druck stets grösser bleibt als der intrathorakale. Am schönsten kommt dies beim spontanen Husten des Pferdes zum Ausdruck; selbst wenn der intrapulmonale Druck einen hohen positiven Wert, ca. $+200 \mathrm{~mm} \mathrm{Hg}$ erreicht, bleibt der intrathorakale negativ (siehe Fig. 2). Nicht die plötzlichen, sprungweisen Änderungen des intrapulmonalen Drucks ziehen eine Änderung des intrathorakalen nach sich, sondern nur die länger dauernden. Bei Wiehern, einer

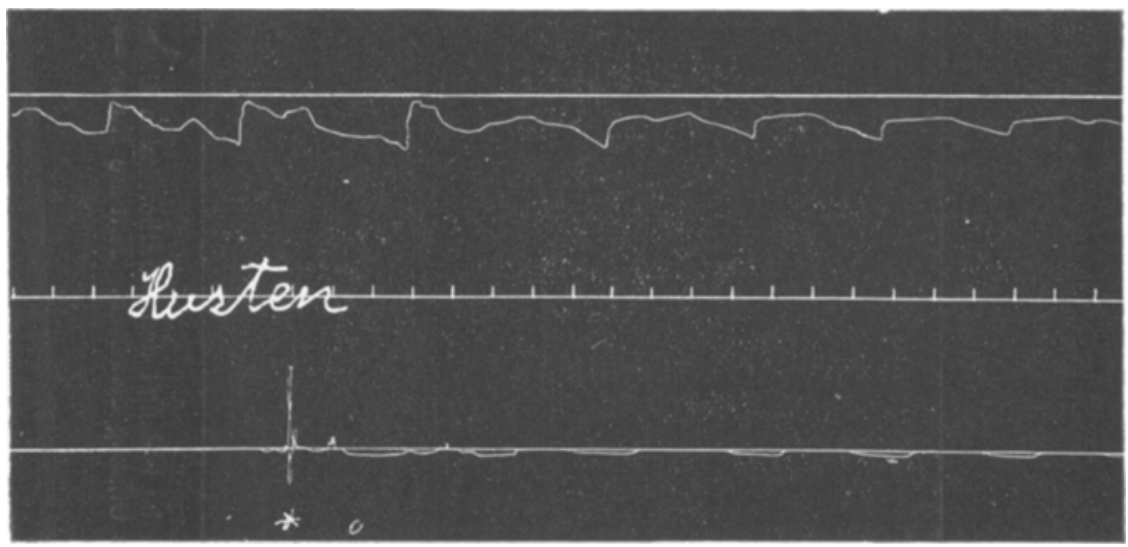

Fig. 2. Intrathorakaler Druck heim spontanen Husten. Obere Kurrenreihe: intrathorakaler Druck; untere: intrapulmonaler; mittlere: Zeit in Sekunden. Bei * Husten.

langgezogenen, forcierten Exspiration wird der intrathorakale Druck für kurze Zeit positiv (siebe Versuch I); bei einem einmaligen, kurzen Hustenstoss dagegen nicht.

Dieser an einem älteren Pferde vorgenommene Versuch hat des weiteren die bemerkenswerte Tatsache ergeben, dass der absolute Wert des intrathorakalen Drucks bei Steigerung der Atemtätigkeit wesentlich hinter dem des jugendlich en Tieres zurückbleibt, obwohl bei älteren Tieren die Absperrung der Atmung lange dauerte. Beim älteren Pferd beträgt er $-300 \mathrm{~mm} \mathrm{Hg}$, beim jüngeren - $500 \mathrm{~mm} \mathrm{Hg}$ (siehe Versuch III). Ausserdem erreicht beim älteren Pferd der Druck im Cavum pleurae später seinen grössten Tiefstand als beim jugendlichen. Bei diesem wird in 50 'Sekunden der Druck von $-500 \mathrm{~mm} \mathrm{Hg}$ erreicht, bei jenem ein solcher von nur $-300 \mathrm{~mm} \mathrm{Hg}$ erst in 72 Sekunden. Hierzu sei ausdrücklich bemerkt, dass in beiden Fällen der Tracheotubus, weleher die Ab- 
sperrung der Atmung vermittelte, absolut fest sass und kein seitliches Einstreichen oder Entweichen der Atmungsluft bei der Inoder Exspiration gestattete,

Es ist deshalb auch gestattet, aus der gefundenen Tatsache den Sehluss zu ziehen, dass für den grösseren Wert des intrathorakalen Drucks beim Fohlen und dem kleineren beim alten Pferd in erster Linie die Elastizität der Lunge verantwortlich zu machen ist, welche bekanntlich in höherem Alter nachlässt. Dyspnöe muss um so früher eintreten, je rascher und je energischer sich die Lunge vermöge ihrer elastischen Kraft zusammenzieht. Es bedeutet demgemäss der mit dem Alter sich einstellende Nachlass der Lungenelastizität innerhalb gewisser Grenzen eine Anpassung an den dyspnoischen Zustand, dem das schwer arbeitende Pferd bekanntermassen unendlich häufig ausgesetzt ist.

Bei demselben Pferde wurde im Verlaufe des Versuchs ein Pneumothorax im linken Pleurasack mittelst eines einem Bistouri caché ähnlichen Instruments erzeugt. Eine Verletzung der Lungen fand, wie die Besichtigung nach dem Tode ergab, nicht statt. Der intrathorakale Druck blieb zunäclıst, solange das Pferd ruhig und oberflächlich nach abdominalem Typus atmete, wenig verändert; er betrug $-80 \mathrm{~mm} \mathrm{Hg}$. Als aber infolge der Absperrung die Atmung sich rasch vertiefte und kostal wurde, erreichte der intrathorakale Druck nach ca. 23 Sekunden während der Exspiration die Abszisse und ging im weiteren Verlauf (37 Sekunden) sogar über diese hinaus bis ca. $+100 \mathrm{~mm} \mathrm{Hg}$. Während der Inspiration betrug er $-190 \mathrm{~mm}$ Hg. Der gleichzeitige intrapulmonale Druck des Pneumothorax wechselte zwischen -110 bei der Inspiration und $+160 \mathrm{~mm} \mathrm{Hg}$ bei der Exspiration (Fig. 3).

\section{Versuch V.}

17. Januar 1910. Älteres, ca, 18 Jahre altes Anatomiepferd, Rappstute. Dieser Versuch bezweckte eine Kontrolle des vorigen unter Schaffung eines gleichseitigen Pneumothorax. Tracheotomiert. Linksseitiger Pneumothorax. Der intrathorakale Druck beträgt anfänglich $-50 \mathrm{~mm} \mathrm{Hg.} \mathrm{Sobald} \mathrm{die} \mathrm{Atmung} \mathrm{sich}$ vertieft, erreicht er während der Exspiration die Abszisse. Absolut negativ ist der Druck nur noch während der Inspiration; hier erreicht er einen Wert von $-130 \mathrm{~mm} \mathrm{Hg}$ bis $-250 \mathrm{~mm} \mathrm{Hg}$ im Maximum.

Während der Exspiration ist der intrathorakale Druck nur noch in bezug auf den intrapulmonalen negativ, insofern er während dieser Phase der Atmung $\pm 0 \mathrm{~mm} \mathrm{Hg}$ und der intrapulmonale $-110 \mathrm{~mm} \mathrm{Hg}$ bis $-220 \mathrm{~mm} \mathrm{Hg}$ im Maximum beträgt. Das Resultat ist somit dasselbe wie im vorigen Versuch. (Fig. 4.) 


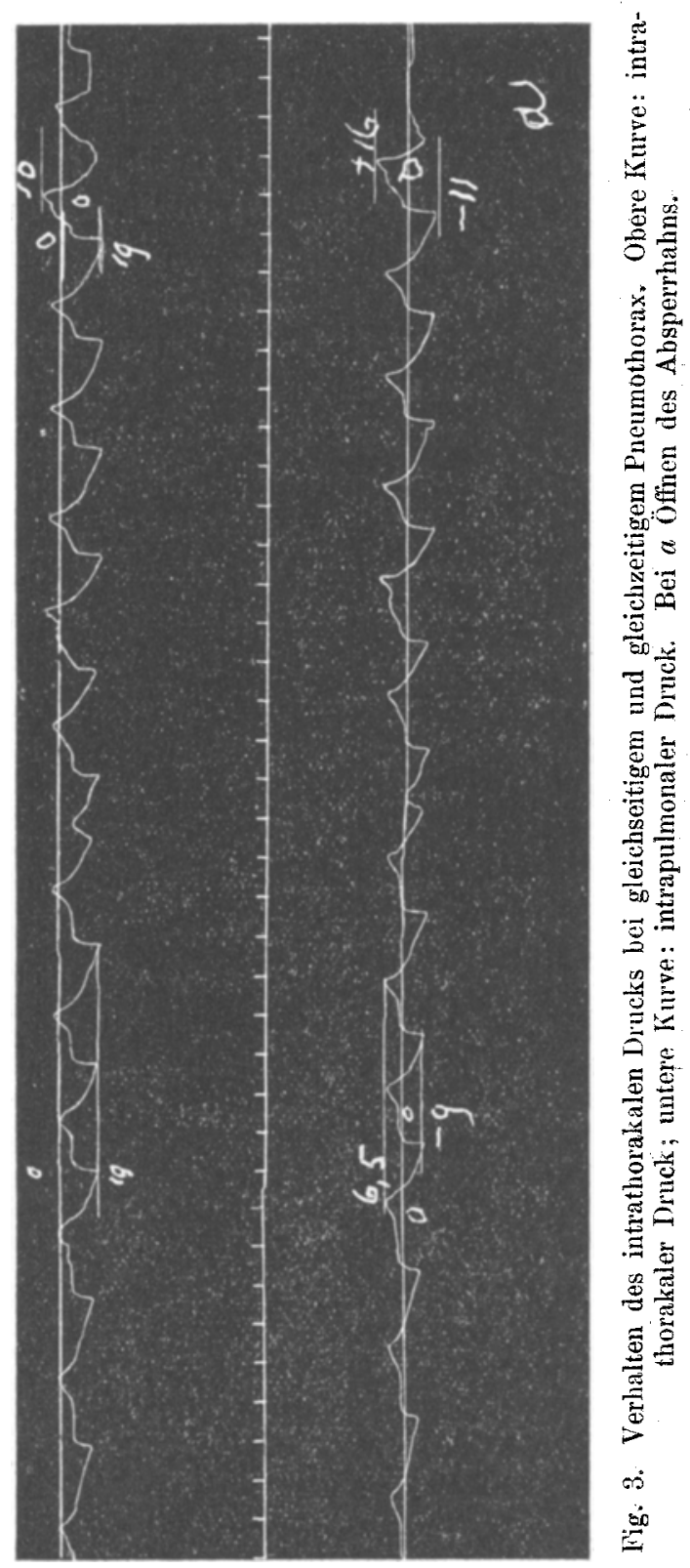



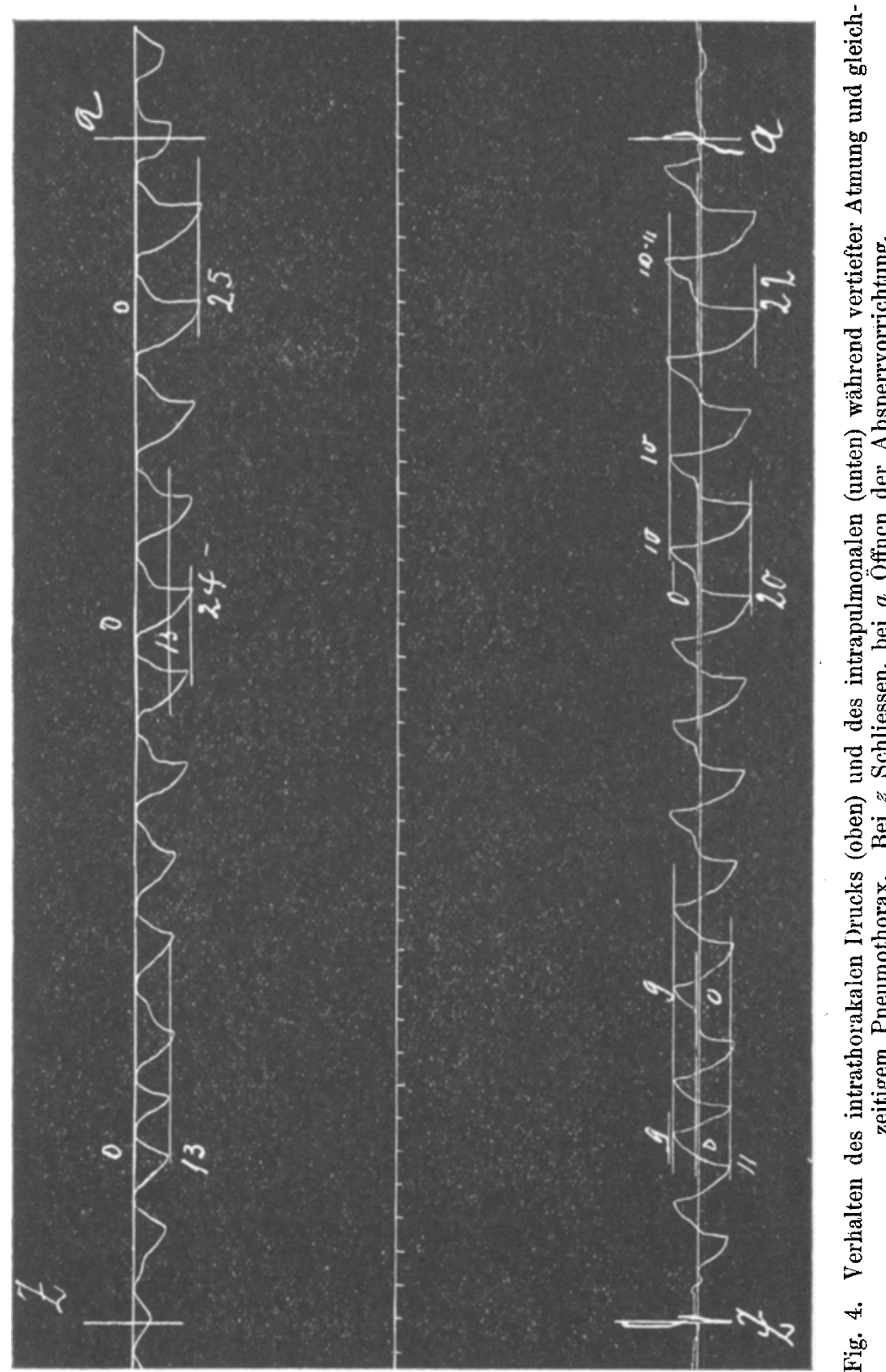


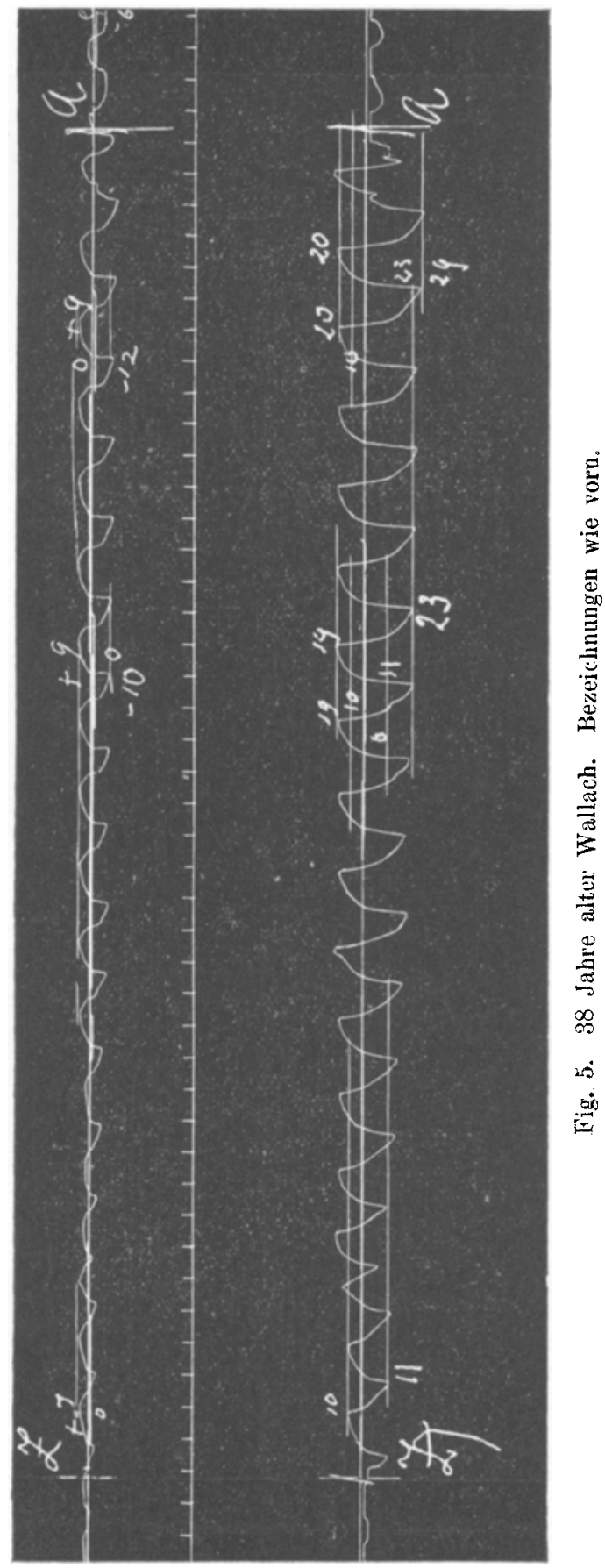




\section{Versnch VI.}

18. Februar 1910. 38. Jahre alter Wallach ${ }^{1}$, tracheotomiert. Einstichstelle links, Pneumothorax links. Das Pferd ist sehr erregt infolge zu starker Kokaininjektion.

Pneumothorax links : Bei normaler A tmung intrathorakaler Druck - $50 \mathrm{~mm} \mathrm{Hg}$ bis $-80 \mathrm{~mm} \mathrm{Hg}$ während der. Inspiration. Bei vertiefter Atmung wird der intrathorakale Druck alsbald positiv und beträgt bei der Exspiration $+70 \mathrm{~mm} \mathrm{Hg}$ bis $+90 \mathrm{~mm} \mathrm{Hg}$; während der Inspiration bleibt er negativ - $100 \mathrm{~mm} \mathrm{Hg}$ bis $-120 \mathrm{~mm}$ Hg. Der intrapulmonale Druck beträgt während der Exspiration $+190 \mathrm{~mm} \mathrm{Hg}$ bis $+200 \mathrm{~mm} \mathrm{Hg}$, bei der Inspiration $-110 \mathrm{~mm} \mathrm{Hg}$ bis $-230 \mathrm{~mm} \mathrm{Hg}$. Somit wird bei Pneumothorax, sobald sich die Atmung vertieft, der intrathorakale Druck positiv, bleibt aber auch hier mit bezug auf den intrapulmonalen regativ, insofern er hinter diesem um 100 bis $130 \mathrm{~mm} \mathrm{Hg}$ zurückbleibt (Fig. 5).

Übersehen wir die vorstehenden Versuche, so lässt sich zunächst feststellen, das der Nachweis des intrathorakalen wie des intrapulmonalen Drucks beim Pferd, sowohl während der Ruhe wie wäbrend der vertieften und angestrengten Atmung möglich ist.

Der intrathorakale Druck beträgt im Mittel -50 bis $-60 \mathrm{~mm}$ Hg. Er schwankt mit der Atmung zwischen $-100 \mathrm{~mm} \mathrm{Ho}$ bei der Inspiration und $-80 \mathrm{~mm} \mathrm{Hg}$ bei der Exspiration. Bei ruhigem Atmen ist er stets negativ. Bei angestrengtem Atmen kann er auch grösser werden als der äussere Luftdruck, bleibt aber immer mit Bezug auf den intrapulmonalen Druck negativ, indem er hinter diesem um ca. $-100 \mathrm{~mm} \mathrm{Hg}$ zurückbleibt.

Bei Fohlen ist wäbrend des ruhigen Atmens der negative Druck etwas kleiner als bei alten Pferden, ca. $-45 \mathrm{~mm} \mathrm{Hg}$ bis $-50 \mathrm{~mm} \mathrm{Hg}$, gegen ca. -50 bis $-70 \mathrm{~mm} \mathrm{Hg}$ bei alten Pferden. Bei Foblen wird aber infolge angestrengter Atmung der negative Druck und zwar in kürzerer Zeit ein grösserer als bei alten Pferden. Es hängt dies mit der Elastizität der Lunge zusammen, die in höherem Alter nachlässt.

Im allgemeinen verlaufen die Schwankungen des intrathorakalen Drucks synehron mit den Schwankungen des intrapulmonalen. Wenn infolge angestrengter Atemtätigkeit der intrapulmonale Druck sich erhöht, so steigert sich auch der durchsehnittliche Minuswert des intrathorakalen Drucks. Während aber der intrapulmonale Druck bei Rückkehr zur normalen Atmung sich rasch ausgleicht, bleibt

1) Das Pferd war nachweislich so alt. 
der negative Druck im Thorax längere Zeit erhöht und kehrt erst allmählich wieder zu seinem Ausgangswert zurück. Kurzdauernden Steigerungen des intrapulmonalen Drucks (z. B. beim Husten) folgt der intrathorakale Druck nicht.

Vorstehende Arbeit wurde im Physiologischen Institut der Tierärztlichen Hochschule in Stuttgart gefertigt. Dem Vorstand des Instituts, Herrn Prof. Dr. Gmelin, sage ich für Überweisung des Themas und für die vielseitjge Unterstützung, besonders bei Anstellung der Versuche, meinen ergebensten Dank. 